\title{
Identification and functional characterization of an alternative splice variant within the fourth exon of human nanog
}

\author{
Jung Sun Kim¹, Jiha Kim, \\ Byung Soo Kim ${ }^{1}$, Hee Yong Chung ${ }^{2}$, \\ Young Yiul Lee ${ }^{3}$, Choon-Sik Park', \\ Young Seek Lee ${ }^{1}$, Young Han Lee ${ }^{1}$ \\ and II Yup Chung ${ }^{1,5}$ \\ ${ }^{1}$ Division of Molecular and Life Sciences \\ College of Science and Technology \\ ${ }^{2}$ Department of Microbiology, College of Medicine \\ Hanyang University \\ Seoul 133-791, Korea \\ ${ }^{3}$ Department of Internal Medicine \\ Hanyang University Hospital \\ Seoul 133-792, Korea \\ ${ }^{4}$ Genome Research Center for \\ Asthma and Allergic Diseases \\ Division of Allergy and Respiratory Medicine \\ Soonchunhyang University Hospital \\ Seoul and Bucheon, Korea \\ ${ }^{5}$ Corresponding author: Tel, 82-31-400-5514; \\ Fax, 82-31-419-1760; E-mail, iychu@hanyang.ac.kr
}

Accepted 21 November 2005

Abbreviations: $\mathrm{BM}$, bone marrow; $\mathrm{CB}$, cord blood; EMSA, electrophoretic mobility shift assay; ES cells, embryonic stem cell; HD, homeodomain; MSC, mesenchymal stem cell; WR, tryptophan-repeat

\begin{abstract}
Nanog, a homeodomain (HD) transcription factor, plays a critical role in the maintenance of embryonic stem (ES) cell self-renewal. Here, we report the identification of an alternatively-spliced variant of nanog. This variant lacked a stretch of amino acids (residues 168-183) located between the HD and tryptophan-repeat (WR) of the previously-reported full length sequence, suggesting that the deleted sequence functions as a linker and possibly affects the flexibility of the $\mathrm{C}$-terminal transactivation domain relative to the DNA binding domain. Expression of mRNA encoding the splice variant, designated as nanog-delta 48, was much lower than that of the full length version in human ES cells. The ratio of nanog-delta 48 transcript to full length transcript increased, however, in multipotent adult progenitor cells. EMSA analysis revealed that both forms of Nanog
\end{abstract}

were able to bind a nanog binding sequence with roughly the same affinity. A reporter plasmid assay also showed that both variants of nanog modestly repressed transactivation of gata-4, whose expression is proposed to be inhibited by nanog, with comparable potency. We conclude that, despite the difference in primary structure and expression pattern in various stem cells, the alternatively-spliced variant of Nanog has similar activity to that of the full length version.

Keywords: alternative splicing; totipotent stem cells; GATA4 transcription factor; hematopoietic stem cells; NANOG protein human

\section{Introduction}

Nanog is a divergent homedomain (HD) transcriptional factor that functions to maintain self-renewal of embryonic stem (ES) cells (Mitsui et al., 2003; Chambers et al., 2003). Forced expression of Nanog confers the undifferentiated state of ES cells independently of the LIF/Stat3 pathway, whereas targeted disruption of the nanog gene or removal of nanog expression results in loss of pluripotency, extra-embryonic endoderm differentiation, recovery of cytokine dependence, and multilineage differentiation. Nanog expression is restricted to pluripotent tissues and ES cell lines and is dramatically reduced by retinoic acid-induced differentiation. In mouse embryos, nanog mRNA is detectable as early as the morula stage, is prominent in the inner cell mass of the blastocyst and epiblast, and is down-regulated by the implantation stage. Blast search of human dbEST reveals that nanog mRNA expression is found in NT2 human teratocarcinoma cells, germ cell and testis tumors, marrow, and other tumors (Cham bers et al., 2003), but not in neural or hematopoietic stem cells (Ramalho-Santos et al., 2002).

The human nanog gene consists of four exons and maps to chromosome $12 \mathrm{p} 13$, along with two other genes (stellar and gdf3) whose expression is restricted to pluripotent cells (Clark et al., 2004). Human and mouse Nanog consist of three parts: an $\mathrm{N}$-terminal region rich in Ser, Thr, and acidic residues; a $\mathrm{HD}$; and a $\mathrm{C}$-terminal region. The $\mathrm{C}$ terminal region contains two transactivation domains, a tryptophan repeat (WR) and a transactivation domain (CD2), and a linker (CD1) connecting HD and WR (Pan and Pei, 2005). Both WR and CD2 
have been shown to separately transactivate a promoter, including a putative Nanog-binding element, while inclusion of CD1 negatively regulates WRmediated transactivation. Unlike its murine counterpart, transactivation activity of human Nanog is confined to its C-terminal region (Oh et al., 2005).

Here, we report an alternatively-spliced form of nanog whose gene product lacks a stretch of 16 amino acid residues located between HD and WR in the full length sequence. The splice variant is similar to its full length version in its ability to bind a Nanog-binding element and transactivate the gata-4 promoter, a proposed transcriptional target of Nanog.

\section{Materials and Methods}

\section{Cell cultures}

Human ES cells were maintained in $80 \%$ Dulbecco's modified essential medium (DMEM)/ F12 supplemented with $20 \%$ KNOCKOUT serum replacement, 0.1 $\mathrm{mM}$ nonessential amino acids (Invitrogen, Carlsbad, CA), $0.1 \mathrm{mM} \beta$-mercaptoethanol (Sigma, St. Louis, $\mathrm{MO}$ ), and $4 \mathrm{ng} / \mathrm{ml}$ human basic fibroblast growth factor (bFGF) (R\&D systems, Inc., Minneapolis, $\mathrm{MN})$. Human ES cells were grown on feeder cells inactivated with mitomycin C (Sigma) in $0.1 \%$ gelatinized tissue culture dishes. Cells were detached by treatment with $1 \mathrm{mg} / \mathrm{ml}$ collagenase IV (Invitrogen) for $30 \mathrm{~min}$ at $37^{\circ} \mathrm{C}$ and then seeded onto new feeder layers once a week. All human cells used in this study were isolated from human subjects that previously gave informed consent. Human umbilical cord blood (CB) was centrifuged through a Ficoll-Hypaque cushion (Sigma), and mononuclear cells collected at the interface and were washed with PBS. These were subjected to RNA isolation or induced to differentiate in IMDM medium containing 10\% FBS (Invitrogen) and 1\% penicillin/streptomycin (Invitrogen) in the presence of IL-3 $(10 \mathrm{ng} / \mathrm{ml})$, IL-5 (10 ng/ml), and GM-CSF (10 $\mathrm{ng} / \mathrm{ml}$ ) (R\&D Systems, Minneapolis, MN) with medium change once a week for 21 days. The CB-derived mononuclear cells were split into CD34and $\mathrm{CD} 34^{+}$subsets using MACS CD34 isolation kit (Miltenyi Biotech, Auburn, CA), according to the manufacturer's instructions. The purity of freshly-isolated $\mathrm{CD}_{3} 4^{+}$cells was between 70 and $80 \%$, as analyzed by FACS (Becton-Dickinson, Mountain View, CA). Human bone marrow (BM) was obtained from healthy donors (ages 10 to $50 \mathrm{yrs}$ ) and its derived mononuclear cells were purified through a Ficoll-Hypaque cushion. To initiate human mesenchymal stem cell (MSC) culture, BM-derived mononuclear cells were cultured in DMEM containing 10\% heat-inactivated FBS, $1 \%$ penicillin-streptomycin, and $2 \%$ glutamine at $37^{\circ} \mathrm{C}$ in $75-\mathrm{cm}^{2}$ tissue culture flask at a density of $2 \times 10^{5} / \mathrm{cm}^{2}$ under $5 \% \mathrm{CO}_{2}$ for 72 h. Non-adherent cells were removed and adherent cells were kept at a density of $1 \times 10^{6}$ cells by changing the medium every 4 days. To assess whether the character of mesenchymal stem cells was maintained during culture, a fraction of the cell cultures was subjected to adipogenic and osteogenic differentiation, as previously described (Meirelles Lda et al., 2003).

\section{RNA isolation, cDNA amplification, and cloning}

Total RNA was isolated with the TRI-Reagent (Molecular Research, Cincinnati, $\mathrm{OH}$ ) and used as template for cDNA synthesis with random hexamers and a reverse transcription kit (Invitrogen). Thirty cycles of polymerase chain reaction (PCR) were performed with an automated thermal cycler, Geneamp PCR system 9700 (Applied Biosystems, Foster City, CA) under the following cycling parameters: denaturation for $30 \mathrm{~s}$ at $95^{\circ} \mathrm{C}$; annealing for $45 \mathrm{~s}$ at $55^{\circ} \mathrm{C}$, and extension for $60 \mathrm{~s}$ at $72^{\circ} \mathrm{C}$. Primers for nanog were as follows: a, 5'-ATGAGTGTGGATCCAGCTTGTCCCCAAAGCTTGCC-3'; b, 5'-TGGCAGAAAAACAACTGGC CG-3'; c, 5'-GTTGCTCCAGGTTGAATTGTTCCAGG-3'; d, 5'-ACACGTCTTVAGGTTGCATGTTCATGGAGTAG-3'. Forward and reverse primers for GAPDH were 5'-CGTCTTCACCACCATGGAGA-3' and 5'-CGGCCATCACGCCACAGTTT-3', respectively. PCR products were analyzed on $1.2 \%$ agarose or $6 \%$ polyacrylamide gel. Full length nanog and its alternatively-spliced variant were subcloned into T-easy vector (Promega, Madison, WI), and the recombinant plasmids were used as templates to confirm the presence of the variants. The cloned sequences were confirmed by an automated DNA sequencer (Applied Biosystems). The coding sequence of human nanog was also cloned into the pcDNA3.1 vector (Invitrogen) for expression in mammalian cells.

\section{Antibody generation and Western blot}

Rabbit anti-human Nanog antibodies were raised against the peptide (residues 168-183) lacking in Nanog-delta 48 (Peptron Inc, Korea). Cells were lysed in 20 mM HEPES, pH 7.2, 1\% Triton X-100, $10 \%$ glycerol, $150 \mathrm{mM} \mathrm{NaCl}, 10 \mu \mathrm{g} / \mathrm{ml}$ leupeptin, and $1 \mathrm{mM}$ PMSF. Protein samples $(20 \mu \mathrm{g})$ were separated by $10 \%$ SDS-polyacrylamide gel electrophoresis and transferred to nitrocellulose filters. Blots were incubated with primary antibodies and developed using an enhanced chemiluminescence detection system (Amersham Pharmacia Biotech., Piscataway, NJ) as previously described (Shin et al., 2004). 


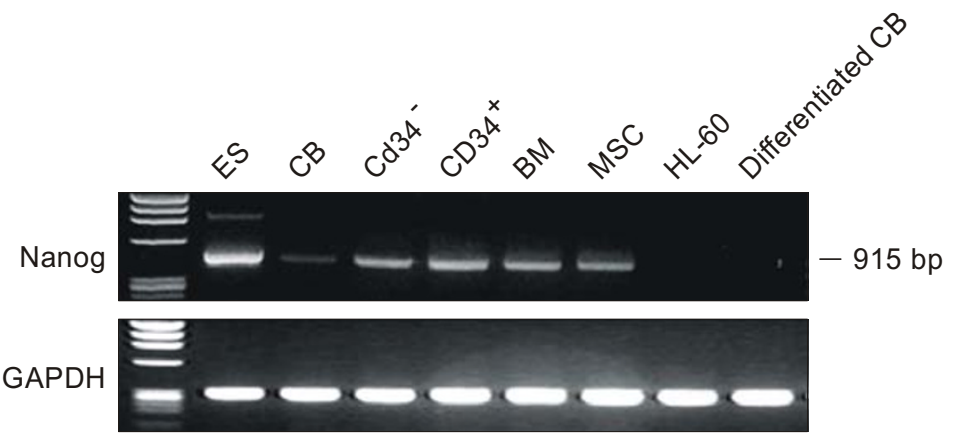

Figure 1. Expression of human nanog transcript. cDNA was synthesized from $4 \mu \mathrm{g}$ of total RNA, amplified using primer pair a and $\mathrm{d}$, as indicated in Figure $2 \mathrm{~B}$, and resolved on a $1.2 \%$ agarose gel. Arrow indicates expected size of PCR product. ES, human embryonic stem cells; $\mathrm{CB}$, human cord blood-derived mononuclear cells; $\mathrm{CD} 34^{+}$ and $\mathrm{CD} 34^{+}$cells fractionated from CB cells by MACS; BM, bone marrow cells; MSC, mesenchymal stem cells; HL60, human promyelocytic leukemia cell line; differentiated CB, $C B$ cells differentiating for 21 days in the presence of IL-3, IL-5, and GM-CSF.

\section{EMSA}

293T cells were transfected with pcDNA3.1 vectors containing nanog or nanog-delta 48 . Fifteen $\mu \mathrm{g}$ of nuclear extracts were solubilized in buffer containing $50 \mathrm{mM}$ HEPES, $400 \mathrm{mM} \mathrm{KCl}, 10 \%$ glycerol, $0.1 \mathrm{mM}$ EDTA, $1 \mathrm{mM}$ DTT, $0.5 \mathrm{mM}$ PMSF, $10 \mu \mathrm{g} / \mathrm{ml}$ leupetin, and $10 \mu \mathrm{g} / \mathrm{ml}$ aprotinin and incubated in $10 \mu \mathrm{l}$ reactions containing $10 \mathrm{mM}$ Tris- $\mathrm{HCl}, 1 \mathrm{mM}$ DTT, $1 \mathrm{mM}$ EDTA, $10 \%$ glycerol, $0.1 \%$ Triton $\mathrm{X}-100,50 \mu \mathrm{g} / \mathrm{ml}$ poly (dl-dC), $0.1 \mathrm{mg} / \mathrm{ml} \mathrm{BSA}$, and $50 \mathrm{mM} \mathrm{KCl}$ for 5 min with or without unlabeled oligonucleotide probes. A ${ }^{32}$ P-labeled wild type probe (5'-CACCCTCAGCCATTAACCTTTTTA-3') or a mutant probe (5'-CACCCTCAGCCAGGAACCTTTTTTA-3') was added to the reaction mixture and incubated for $20 \mathrm{~min}$. For supershift assay, anti-human Nanog or pre-immune rabbit serum was pre-incubated with the mixture for $30 \mathrm{~min}$ before incubation with labeled probe. Reaction products were resolved on a $5 \%$ polyacrylamide gel and visualized by autoradiography.

\section{Transfection, construction of promoter, and reporter assay}

The gata-4 promoter, including 1117 bases upstream from the transcription start site and the 5'-UTR of gata-4, was PCR-amplified with a forward primer, 5'-CAAAACGCAGGATGCTGGGAAG-3', and a reverse primer, 5'-GGCCAAGCTCTGATACATGGTC -3'. The PCR product was cloned into the pGL3basic vector, a luciferase reporter plasmid. 293T cells were plated at a density of $5 \times 10^{5}$ cells $/ 35 \mathrm{~mm}$ dish and cotransfected with $2 \mu \mathrm{g}$ of the reporter plasmid and various concentrations of nanog-expressing vector in triplicate sets using lipofectamin 2000, according to the manufacturers' instruction (Invitrogen). The total amount of nanogexpressing vector and empty plasmid (pcDNA3.1) was adjusted to $2 \mu \mathrm{g}$.

For the luciferase assay, cell were harvested and lysed in 1x PLB buffer (Promega). Luciferase activity was measured with $2 \mu \mathrm{g}$ of total protein using a TD2020 luminometer (Berthold, Germany). Fold induction was calculated as the ratio of gata- 4 reporter plasmid to pGL3basic vector. A MannWhitney $U$ test was applied to compare differences between nanog and nanog-delta 48 , with the results expressed as mean \pm standard error of the mean (SEM).

\section{Results and Discussion}

Expression of the nanog gene was examined in several multipotent progenitor cells by RT-PCR. As analyzed by agarose gel electrophoresis, nanog transcripts were abundant in human embryonic stem (ES) cells (Figure 1). The nanog transcript was also expressed in several multipotent adult progenitor cells, including $\mathrm{CB}$ cells (both $\mathrm{CD} 34^{-}$and $\mathrm{CD} 34^{+}$ cells from CB cells), BM cells, and MSCs, although the expression levels in these cells were much lower than in ES cells. The transcript was not detectable in CB cells differentiated in the presence of IL- $3, \mathrm{IL}-5$, and GM-CSF for 21 days or in HL60 promyelocytic leukemia cell line.

We amplified full length nanog using primers a and $\mathrm{d}$ (Figure 2B), and then cloned and sequenced it. The expected size was $915 \mathrm{bp}$, corresponding to a polypeptide of 305 amino acid residues. Of 18 clones sequenced, the nucleotide sequences of 16 were identical to the published sequence of the nanog gene (GenBank accession no. NM024865), except for a few sequence variations (C381T, A492G, C747T, and C1014T) that did not cause amino acid substitutions. The remaining two clones were missing $48 \mathrm{bp}$ at the beginning of the fourth exon of the gene (Figure 2A) and designated as nanog-delta 48 (GenBank accession no. AY578089). Analysis of this deleted sequence revealed the presence of a polypyrimidine tract followed by $A G$ at the 3 ' end, suggesting that nanog-delta 48 had been generated by alternative splicing. To confirm the existence of the two splice variants, we carried out RT-PCR with total RNA from ES cells and compared these sequences with those of the PCR-amplified 
A

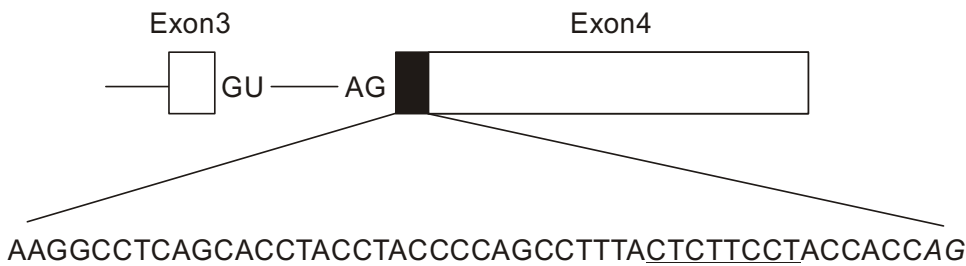

B

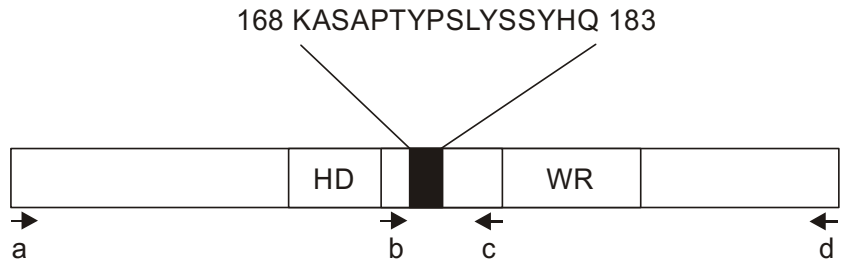

C
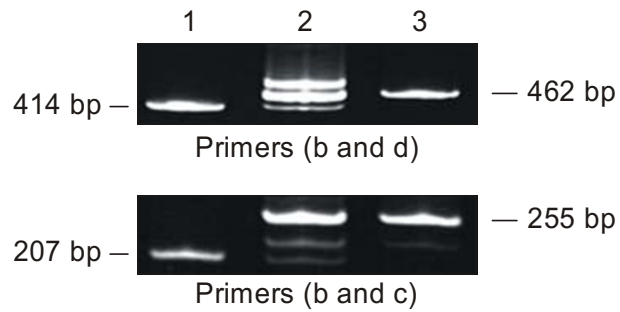

D

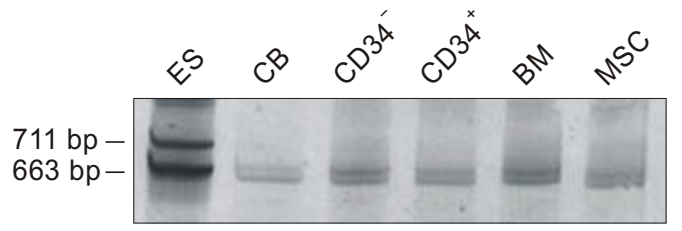

Figure 2. Alternatively-spliced variant of human nanog. (A) A partial structure of the nanog gene that covers exon 3, exon 4 , and an intron between them. The sequence of 48 nucleotides (black box) at the beginning of exon 4 that is removed by alternative splicing contains a polypyrimidine tract (underlined) and a $3^{\prime}$ splice site at the end of the sequence (italic). (B) Nanog protein structure. The deleted region (black box) resulting from the alternative splicing is indicated, as are the HD and WR domains and its amino acid sequence (residues 168-183) is also indicated. Arrows represent the positions of primers for RT-PCR. (C) Identification of alternatively-spliced transcripts of nanog. cDNA that had been prepared from ES cells was amplified using the primer sets indicated (lane 2). The alternatively-spliced transcript (nanog-delta 48) and the full length version were cloned, sequenced, and used as templates for PCR amplification (lanes 1 and 3 , respectively). The amplified products were run on a sequencing gel. The bands that did not comigrate with the PCR products of cloned nanog variants were nonspecific. (D) Relative abundance of nanog and nanog-delta 48 transcripts. RT-PCR was carried out using primer pair a and $\mathrm{C}$, as indicated in Figure 2B. Arrows indicate amplicons of $711 \mathrm{bp}$ and $663 \mathrm{bp}$ corresponding to nanog and nanog-delta 48 , respectively, resolved on a sequencing gel. $\mathrm{CB}$, human cord blood-derived mononuclear cells; $\mathrm{CD}_{3} 4^{+}$and CD34 cells fractionated from CB cells by MACS; BM, bone marrow cells; MSC, mesenchymal stem cells. products using the two cloned nanog variants as templates with two different primer pairs, b and d, and $\mathrm{b}$ and $\mathrm{c}$, as indicated in Figure $2 \mathrm{~B}$. As resolved on a sequencing gel, RT-PCR with each primer pair gave rise to three different products. Two of these co-migrated as the PCR products from the corresponding cloned nanog variants (Figure $2 \mathrm{C}$ ).

The same sequence gap of $48 \mathrm{bp}$ reported here has also been reported from several EST clones derived from ES cells (GenBank accession no. CD642640), adult bone marrow (GenBank accession no. BF893623 and BF900155), and epid tumor cells (GenBank accession no. BF773088). It has also been shown that exon 4 lacking the 48 bp is present in one (nanogp1) of human nanog pseudogenes (Booth and Holland, 2004).

After isolating the two splice variants of nanog, we examined their relative abundance. The transcript of full length nanog was expressed in ES cells to a much greater extent than was that of nanog-delta 48 (Figure 2D). Heightened expression of the full length transcript over the nanog-delta 48 transcript was not observed, however, in multipotent adult progenitor cells. Rather, relative expression of the nanog-delta 48 transcript to the full length nanog transcript was considerably increased as compared with ES cells.

It has been reported that the relative proportion of two different transcripts of a gene resulting from alternative splicing varies during development. This study, by Takeda et al., demonstrated that pou-2 is a full length transcriptional regulator expressed in zebrafish from the one-cell stage until formation of the gastrula and that a shorter, alternatively-spliced variant, t-pou2, is increased in the early gastrula and declines in the neurula stage. Furthermore, overexpression of the variant in the embryo was shown to cause developmental retardation or arrest. The relative abundance of t-pou2 to pou2 transcript increases in early gastrula and declines in neurula stage (Takeda et al., 1994). It is shown that downregulation of nanog is correlated with ES cell differentiation from early blastocyst to peri-implantation and, thus, loss of pluripotency (Chambers et al., 2003; Mitsui et al., 2003). Hence, the expression 
level of nanog is a critical factor to determine pluripotency. Given that nanog expression occurs during the blastocyst stage, which is believed to not yet be committed to a particular fate (Cavaleri and

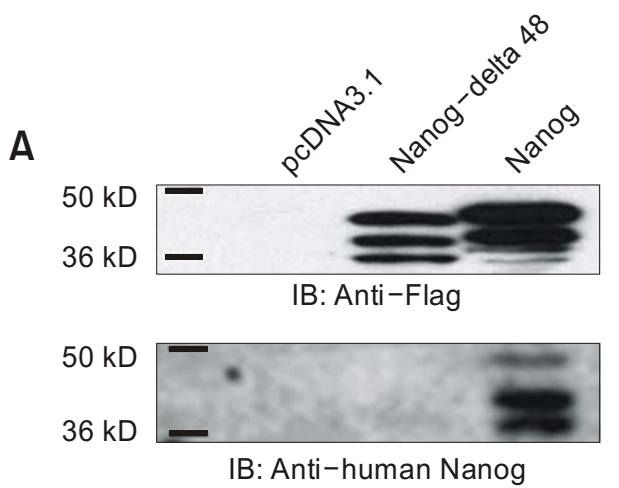

B

\begin{tabular}{|llll}
1 & 2 & 3 & 4 \\
\hline & & &
\end{tabular}

Figure 3. Nanog protein expression. (A) Nanog and nanog-delta 48 were transiently transfected into 293T cells and their expression was analyzed by immunoblotting (IB) with anti-Flag or anti-human Nanog antibodies. (B) 293T cells were transiently transfected with nanog and harvested at $0 \mathrm{~h}$ (lane 1), $24 \mathrm{~h}$ (lane 2), $48 \mathrm{~h}$ (lane 3), and $72 \mathrm{~h}$ (lane 4) post-transfection.
Scholer, 2003), the increase in the ratio of nanogdelta 48 to full length nanog expression in cells of lesser pluripotency suggests that nanog-delta 48 may play a role in the fine control of differentiation. Consequently, it will be worthwhile to investigate the relative expression of nanog and its splice variant during differentiation of ES cells. Availability of the variant-specific antibody will help to clarify the physiologic relevance of the shorter protein.

As the sequence of $48 \mathrm{bp}$ was deleted in frame, the alternative splicing did not alter a reading frame or introduce amino acid substitutions. The amino acid sequence (residues 168-183) corresponding to the deleted nucleotide sequence was positioned between those nucleotides encoding the HD and WR domains (Figure 2B), indicating that the deleted sequence acts as a linker to connect the two functionally important domains. Additionally, the deleted region largely consisted of hydrophilic amino acids. A comparison of deduced amino acid sequence revealed that the deleted region had $68 \%$ sequence identity to the corresponding region of mouse Nanog, greater sequence homology than is observed over the entire Nanog sequence (58\%).

When nanog and nanog-delta 48 constructs with Flag at their C-termini were transiently transfected into 293T cells, three protein species were detected. These included two protein species of low molecular mass that were considered to be degradation products of the largest one (Figure $3 \mathrm{~A}$ ). Nanog-delta 48 migrated, as expected, due to the missing sixteen
A

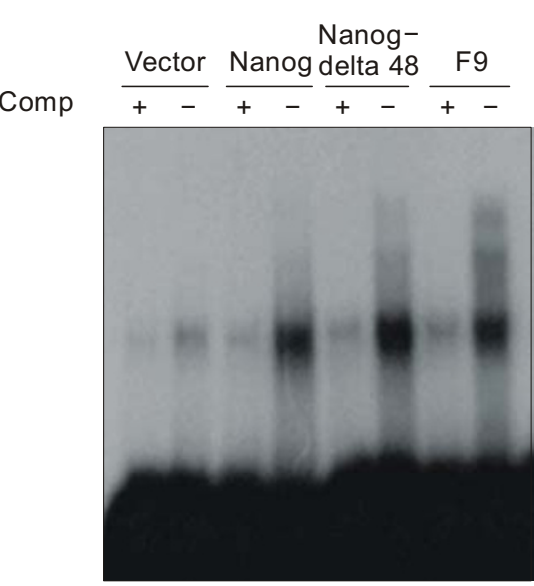

B

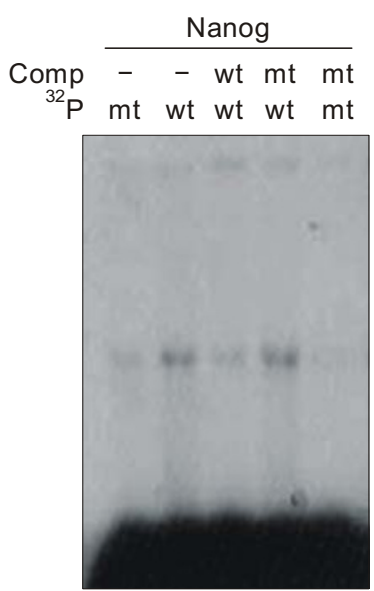

wt: CACCCTCAGCCATTAACCTTTTTA mut: CACCCTCAGCCAGGAACCTTTTTA

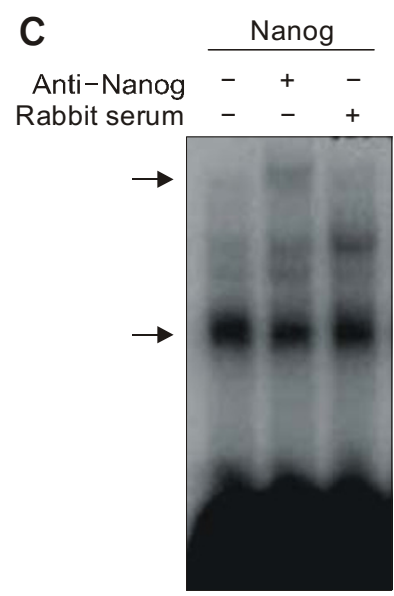

Figure 4. DNA binding of Nanog and Naog-delta 48. 293T cells were transiently transfected with vector (pcDNA3.1), nanog, or nanog-delta 48. (A) Nuclear extract was prepared and incubated with ${ }^{32} \mathrm{P}$-labeled probe. Competitor (Comp) was in 50 -fold excess. Nuclear extract from F9 cells was used as a positive control. (B) Nuclear extract from Nanog-expressing transfectant was incubated with either wild-type (wt) or mutant (mt) probe. Competitor was in 50-fold excess. (C) Nuclear extract from 293T cells transfected with nanog was pre-incubated with anti-human Nanog or normal rabbit serum for $1 \mathrm{~h}$ prior to incubation with labeled probe. Arrow indicates a probe-protein complex, while arrow head indicates a supershift by the addition of anti-human Nanog. 
residues, with a slightly smaller molecular mass than the full length version. Nanog-delta 48 was not reactive to a polyclonal antibody raised against the deleted sequence of 16 amino acids otherwise present in Nanog. Nanog protein was expressed at 24 to $48 \mathrm{~h}$ but disappeared at $72 \mathrm{~h}$ post-transfection (Figure 3B).

EMSA analysis showed that Nanog-delta 48 bound a putative Nanog-binding sequence with apparently equal affinity as the full length version (Figure 4A), indicating that deletion of the putative linker region in Nanog does not affect its DNA-binding capability. In addition, the nuclear extract from $\mathrm{F} 9$ cells, which are murine embryonic teratocarcinoma cells and constitutively expressed nanog mRNA (data not shown), bound the same Nanog-binding sequence as did human Nanog. The binding was specific, as a mutant nanog probe did not form a protein-DNA complex (Figure 4B). A supershift was observed with anti-human Nanog antibody (Figure 4C).

Pan and Pei demonstrated that Nanog has at least two transactivation domains (Pan and Pei, 2004), with both the $\mathrm{N}$ - and $\mathrm{C}$-termini relative to $\mathrm{HD}$ (residues 95-154) having a transactivation domain. They further showed that the C-terminal region, corresponding to residues 156 to 305 , contains, sequentially, CD1, a region between $H D$ and WR, and two transactivation domains, WR and CD2. WR or CD2 individually function as transctivators, while CD1 alone does not act as a transctivator but rather inhibits WR-mediated transactivation (Pan and Pei, 2005). It remains unknown, however, whether and how these domains function in the transcriptional regulation of real target genes of Nanog, because the transctivation activity was determined using GAL4-DBD fusion and reporter system, such as artificial promoters containing Nanog-binding elements singly or in tandem. The region that is removed by alternative splicing (residues 168-183) is within the CD1. This deleted region did not show any preferred secondary structure when analyzed by known prediction programs (www.expasy.ch), suggesting that it may serve as a linker between the HD and the C-terminal transactivation domain.

As Nanog has been proposed to act as a transcriptional repressor for the gata-4 gene, we tested whether the splice variants would differentially modulate the gata-4 promoter (Mitsui et al., 2003). We constructed a reporter plasmid that included the region spanning 1117 upstream of the gata-4 promoter and the 5 'UTR of gata- 4 . The gata- 4 promoter construct included five ATTA sequences, a key nucleotide sequence for potential binding of HD-containing transcription factors. When individual sequences including the ATTA element were compared with the published consensus sequence of the
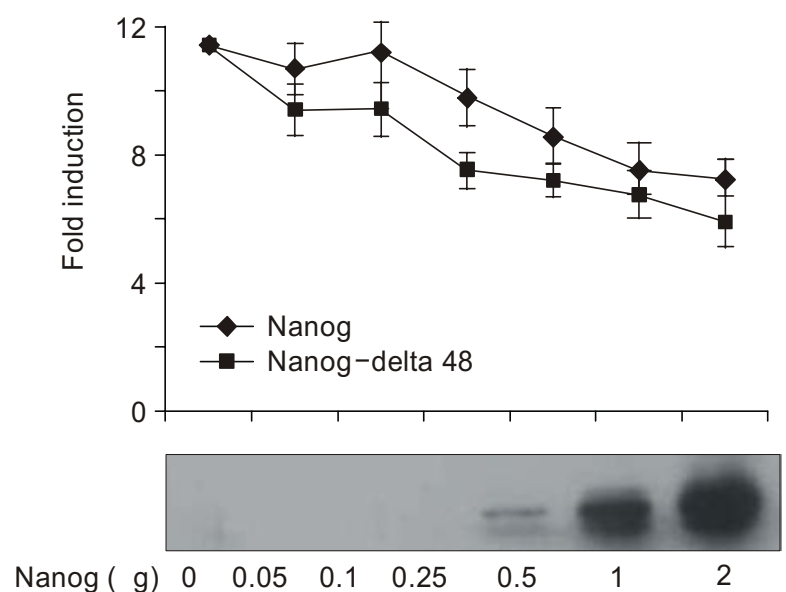

Figure 5. Transactivation of gata-4 promoter. A reporter plasmid in which the gata-4 promoter is linked to a luciferase reporter was cotransfected into 293T cells with increasing concentrations of nanog or nanog-delta 48 . After $36 \mathrm{~h}$, cell extracts were prepared and subjected to luciferase assay. Data are presented as the average of four independent experiments. Nuclear extracts from nanog (bottom panel)-transfected 293T cells were prepared from an aliquot of cells and subjected to Western blot analysis. Expression of Nanog-delta 48 was similar to that of Nanog (data not shown)

Nanog binding motif, (C/G)(G/A)(C/G)C(G/C)ATTAA $(\mathrm{G} / \mathrm{C})$, the sequence TCTGCATTAAC at -572 of the gata-4 promoter was the most similar. When the reporter plasmid was cotransfected into 293T cells with nanog- or nanog-delta 48-expressing plasmids, transactivation of the gata-4 promoter was inhibited in a dose-dependent manner, with inhibition reaching $40 \%$ at a concentration of $2 \mu \mathrm{g}$ of expression vector. Nanog-delta 48 tended to inhibit the gata-4 promoter at a slightly higher level than did the full length version, although the difference was not statistically significant (Figure 5). Although our results showed no significant difference in transactivation between Nanog and Nanog-delta 48, further study is required in order to determine whether Nanog and Nanog-delta 48 exhibit inhibitory activity against the gata-4 promoter in a physiological setting. Such a setting might be cells differentiating to endoderm, as in the case of F9 cells. In addition, a conventional promoter deletion experiment is needed to clearly demonstrate gata-4 regulation by Nanog.

\section{Acknowledgement}

This work is supported by grants from KOSEF (R01-2005-000-10728-0) and KISTEP (M10310570003-04N0257-00310). We thank Drs. Ji Yoen Lee, Sang Hun Lee, Yong Sung Lee, and Key-Sun Kim for supply of total RNA from human ES cells and 
helpful discussion. We also thank Dr. Sung Hee Choi, Hanvit Clinic, Ansan for kindly supply of $C B$ cells.

\section{References}

Ando K, Shioda S, Handa H, Kataoka K. Isolation and characterization of an alternatively spliced variant of transcription factor Islet-1. J Mol Endocrinol 2003;31:419-25

Booth HA, Holland PW. Eleven daughters of NANOG. Genomics 2004;84:229-38

Cavaleri F, Scholer HR. Nanog: a new recruit to the embryonic stem cell orchestra. Cell 2003;113: 551-2

Chambers I, Colby D, Robertson M, Nichols J, Lee S, Tweedie S, Smith A. Functional expression cloning of nanog, a pluripotency sustaining factor in embryonic stem cells. Cell 2003;113:643-55

Clark AT, Rodriguez RT, Bodnar MS, Abeyta MJ, Cedars MI, Turek PJ, Firpo MT, Reijo Pera RA. Human STELLAR, NANOG, and GDF3 genes are expressed in pluripotent cells and map to chromosome 12p13, a hotspot for teratocarcinoma. Stem Cells 2004;22:169-79

Meirelles LS, Nardi NB. Murine marrow-derived mesenchymal stem cell: isolation, in vitro expansion, and characterization. Br J Haematol 2003;123:702-11

Mitsui K, Tokuzawa $\mathrm{Y}$, Itoh $\mathrm{H}$, Segawa $\mathrm{K}$, Murakami M, Takahashi K, Maruyama M, Maeda M, Obenauer JC, Cantley LC, Yaffe MB. Scansite 2.0: proteome-wide prediction of cell signaling interactions using short sequence motifs. Nucleic Acids Res 2003;31:3635-41
Oh JH, Do HJ, Yang HM, Moon SY, Cha KY, Chung HM, Kim $\mathrm{JH}$. Identification of a putative transactivation domain in human Nanog. Exp Mol Med 2005;37:250-4

Pan GJ, Pei DQ. Identification of two distinct transactivation domains in the pluripotency sustaining factor Nanog. Cell Res 2003;13:499-502

Pan GJ, Pei DQ. The stem cell pluripotency factor nanog activates transcription with two unusually potent subdomains at its c-terminus. J Biol Chem 2005;280:1401-7

Ramalho-Santos M, Yoon S, Matsuzaki Y, Mulligan RC, Melton DA. "Stemness": transcriptional profiling of embryonic and adult stem cells. Science 2002;298:597-600

Shin SY, Kim CG, Hong DD, Kim JH, Lee YH. Implication of Egr-1 in trigluoperazine-induced growth inhibition in human U87MG glioma cells. Exp Mol Med 2004;36:380-6

Takeda H, Matsuzaki T, Oki T, Miyagawa T, Amanuma H. A novel POU domain gene, zebra fish pou2: expression and roles of two alternatively spliced twin products in early development. Genes Dev 1994;8:45-59

Yaffe MB, Leparc GG, Lai J, Obata T, Volinia S, Cantley LC. A motif-based profile scanning approach for genome-wide prediction of signaling pathways. Nature 2001;19:348-53

Yamanaka S. The homeoprotein Nanog is required for maintenance of pluripotency in mouse epiblast and ES Cells. Cell 2003;113:631-42 